\title{
Parallel Program Debugging with MAD - A Practical Approach
}

\author{
Dieter Kranzlmüller ${ }^{1}$ and Axel Rimnac ${ }^{2}$ \\ ${ }^{1}$ GUP, Dept. for Graphics and Parallel Processing \\ 2 Institute for Theoretical Physics \\ Joh. Kepler University Linz \\ Altenbergerstr. 69, A-4040 Linz/Austria, Europe \\ \{dieter.kranzlmueller, axel.rimnac\}@jku.at
}

\begin{abstract}
Debugging parallel programs can be very time-consuming and tedious, because the multiplicity of communicating processes increases the complexity of a program and the probability of incorrect behavior. Solutions are provided by debugging tools, which try to offer meaningful ways to investigate errors and their original causes. The MAD environment is a debugging toolset, which focuses on parallel and distributed programs. This paper discusses the application of MAD to real-world programs with practicability and usability as the main goals. The observations are based on a series of debugging sessions conducted for a specific application of theoretical physics, with the opinions of the tool developer on one side and the view of the application developer on the other side.
\end{abstract}

\section{Introduction}

Computational Science and Engineering (CSE) is strongly related to High Performance Computing (HPC), because HPC promises the performance needed by many CSE applications. The power of HPC architectures is usually provided by parallel and distributed systems, which represent a serious challenge for CSE application developers. The traditional software engineering process has to be adapted to cope with several concurrently executing and communicating processes, which attempt to jointly solve a given problem. This increases the complexity of parallel and distributed CSE applications and requires dedicated support from software development tools.

A basic necessity for software is correctness, which is verified during the testing and debugging phase of the software lifecycle. The program's behavior during runtime is inspected and compared to the given specification. When deviations from the specified behavior are observed, the developer tries to locate the original cause for the anomaly and eventually corrects the program's code. These activities are supported by so-called debugging tools, which provide techniques such as breakpointing and inspection for program comprehension [12].

Although many parallel debuggers exist, the majority of error detection work is still performed by placing "print" statements in the program and inspecting their output during execution. This seems almost archaic compared to the functionality provided by sophisticated debugging tools, such as DDBG [4], P2D2 [7], DETOP [13], and Totalview [5]. 
One reason for this situation may be that placing print statements is rather trivial compared to the learning curve of using a new debugging tool. Another reason may be that the textual representations chosen by the majority of debugging tools may be inappropriate to express the complexity of parallel and distributed programs [11]. Finally, there may be a discrepancy between the views of the tool developer and the application programmer on what is important about parallel debugging, or rather how and what the debugging tool should support.

This paper describes a case study of parallel program debugging for a particular computational science program. The idea was to investigate the usefulness of an existing debugging tool when developing a theoretical physics program. The approach was rather informal, with the two persons involved sitting together and detecting errors. Consequently, the following article contains an example set of errors detected during the course of debugging. Nevertheless, the findings of this cooperation seem to be an interesting point for a discussion about the practical usefulness of software development tools.

The paper is organized as follows. The next section describes the case study, an application from theoretical physics, and the MAD environment. Section 3 introduces the required preparations for error detection and presents simple automatic debugging features, which revealed several errors in the program. Section 4 shows some more advanced debugging features by using array visualization, while a summary and an outlook on future work conclude this paper.

\section{Case Study: Debugging with the MAD Environment}

\subsection{Target Application}

The application chosen as a target for analyzing the debugging process under "real world" conditions is a program that is currently used in the investigation of the scattering of single helium $\left({ }^{4} \mathrm{He}\right)$ particles on a macroscopic slab system consisting of ${ }^{4} \mathrm{He}$ particles near absolute zero temperature. Helium near absolute zero is known to be the only terrestrical liquid that shows purely quantum mechanical effects. Hence, the description of scattering has to be done in the framework given by the well-known Schrödinger equation. Therefore, the quantity containing maximum information on the system is the quantum mechanical wave function of the given many-body system.

The calculations are done in the so-called Correlated Basis Function (CBF) approximation which describes the energy loss of an incident particle to the target slab in terms of two-phonon decay processes, i.e., an incident particle may stick in the system by losing its energy to two quasiparticle excitations that can be viewed as surface waves on the system. The incident particle may also lose just part of its energy and leave the slab either as a reflected or transmitted particle1.

The equation for the quantities of major interest, namely the probabilities of elastic reflection and transmission as well as the probability of inelastic scattering takes the form [2]

\footnotetext{
${ }^{1}$ A classical analog would be the process of throwing a small stone into a lake. At this process the stone may be reflected elastically or stick in the liquid and sink.
} 


$$
H_{1}(z) \psi(z)+\int \Sigma\left(z, z^{\prime}, \omega\right) \psi\left(z^{\prime}\right) d z^{\prime}=\hbar \omega \int S\left(z, z^{\prime}\right) \psi\left(z^{\prime}\right) d z^{\prime}
$$

which is a generalized one-dimensional Schrödinger equation. Here, $\psi(z)$ is the quantum mechanical wave function of the incident particle. $H_{1}(z)$ and $S\left(z, z^{\prime}\right)$ depend on the structure of the static background liquid, $\omega$ is the energy of the incident particle and $\Sigma\left(z, z^{\prime}, \omega\right)$ is the so-called nonlocal optical potential accounting for the possibility of the incident particle to lose energy. The self energy matrix $\Sigma\left(z, z^{\prime}, \omega\right)$ is given by

$$
\Sigma\left(z, z^{\prime}, \omega\right)=\sum_{m, n} \int \frac{V_{m n}(p, q, z) V_{m n}\left(p, q, z^{\prime}\right)}{\hbar\left(\omega_{m}(q)+\omega_{n}(p)-\omega\right)} d p d q
$$

where the matrix elements $V_{m n}(p, q, z)$ are known ground state quantities and the denominator displays the dispersion relations of so-called Feynman excitation [3]. In determining $\Sigma$, parallelization is done by data-decomposition along indices $m, n$ and summing up all contributions at the end of the calculation. This is by far the most time-consuming part of the computation.

Practical calculations have revealed that the execution time for a single run is about $12 \mathrm{hrs}$ on 8 processors of an SGI Origin 3800 (equipped with R12000 processors at 450 $\mathrm{MHz}$ ). This clearly justifies the use of a HPC system and parallel software engineering for application development. The program is written in FORTRAN 90 and uses the Message Passing Interface standard MPI [10] for communication and synchronization. The following sections describe the development of the application described above from a debugging point of view.

\subsection{The MAD Environment}

The Monitoring And Debugging environment MAD [8] is a toolset for debugging parallel programs based on the message-passing paradigm. The principal idea of MAD is to display observed program interactions as space-time diagrams and to apply this graphical representation for error detection. Based on this idea, two kinds of errors can be distinguished [9]:

- Local errors represent incorrect program behavior without influence of parallelism. They occur on a single process and do not involve communication or interaction with other processes. These kinds of errors can be investigated with traditional sequential debuggers.

- Global errors are established due to the parallel nature of the application. They are connected to operations which would not exist in sequential programs. As a consequence, the occurrence of incorrect behavior and its original source are often located on different processes. Due to these characteristics, the usability of sequential debuggers is questionable for tracking global errors.

For the remainder of this paper, we will focus on global errors, because their existence is determined by the parallelism constructs introduced when developing programs for an HPC system. Nevertheless, it is obvious that debugging local errors may also be needed, for example when tracing back sources of global errors. 
The debugging process of MAD is performed using a series of independent modules. The two most important modules of MAD are

- the monitoring tool NOPE - NOndeterministic Program Evaluator, and

- the visualization tool ATEMPT - A Tool for Event ManiPulaTion.

While these core modules sufficiently cover the majority of debugging activities, several other analysis tools extend the functionality of MAD. Examples include trace file converters for various formats, additional visualization modules, and a control- and data flow analysis tool [8].

\section{Program Instrumentation and Automatic Error Detection}

The initial step for program debugging is instrumentation, which is applied to insert code containing monitoring functionality into the target program. The monitoring code is then responsible for extracting program state data for subsequent analysis activities. In MAD, monitoring is performed with NOPE, which is inserted into the target program through source code instrumentation. While source code instrumentation is less sophisticated than other techniques, e.g. dynamic instrumentation [6], its simplicity is a major advantage. The drawback with this method is that the source code has to be recompiled in order to integrate the monitoring functionality. However, this is probably acceptable since it does not require more effort than inserting print statements into the program.

With the monitoring tool NOPE, instrumentation is performed in two ways:

(1) Automatic instrumentation is used for all functions known a priori. This includes the library function calls of MPI as well as some system functions. The monitoring code is inserted by replacing the include file mpif. $h$ with its counterpart of NOPE - monitor. $h$ - which replaces the original function calls through preprocessor directives.

(2) Manual instrumentation is required whenever behavior needs to be observed, which is specific to the target application. This includes switching on and off the monitoring functionality as well as monitoring of user-defined data structures. In this case, the user has to insert monitoring functionality in the code in the same way as print statements are used.

For the target application, we applied both kinds of instrumentation. While automatic instrumentation was turned on for the complete debugging session, manual instrumentation required several iterations to extract the state information of interest.

The most important characteristic of instrumentation seems to be simplicity. A debugging tool will only be utilized, if instrumentation and monitoring is less complicated than placing print statements.

After recompiling the program with the monitoring functionality of NOPE included, another program execution is initiated. The state data extracted by NOPE is stored in tracefiles, which can afterwards be analyzed with the visualization tool ATEMPT. Instead of exhaustively discussing the features of MAD, the following paragraphs describe a few situations, that occurred during the debugging sessions of the target application. 
Situation 1: Early in the debugging session, the program hangs for no apparent reason. The diagnosis print statements indicate that the execution of the program is blocked at some point, but does not reveal the exact location in the source code nor the actual reason thereof.

Among the reasons why software hangs, the most typical case is a deadlock due to an unfulfilled condition at a blocking operation. An example from MPI is the blocking receive MPI_Recv, which holds a process' execution until a specific message arrives. If the message does not arrive - for whatever reason - the process' execution is blocked forever. This kind of error is a so-called isolated receive [9].

The problem with this kind of situations is that blocked executions and program failures are difficult to observe. The reason is based on the fact, that the monitoring tool needs to store the monitoring data to disk. Yet, if the operation of a process is blocked, the important information may still be in some intermediate monitoring or file buffer. If the program's execution is terminated, the data from the file buffer is lost.
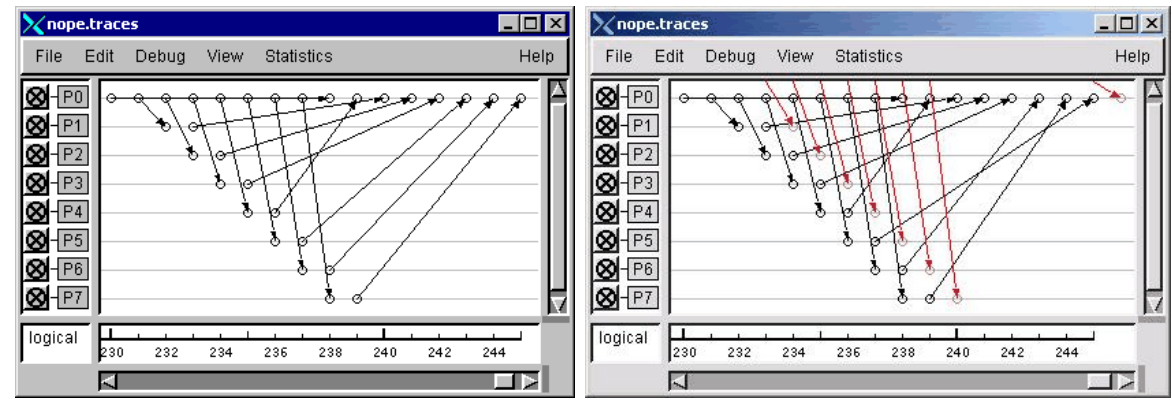

Fig. 1. Missing trace data due to program failure.

An example of this phenomenon is shown in Figure1, The left screenshot displays the program's execution as observed during execution with the ATEMPT visualization tool. The program hangs due to a blocking receive on process $\mathrm{P} 0$, which is actually not shown in Figure 1 Instead, it seems as if the program terminated without any reason.

With NOPE, tracefile flushing can manually be turned on or off at any place during the program's execution. After turning tracefile flushing on and restarting the program, the right screenshot is observed. The program still hangs for the reason mentioned above, but the cause of the hanging is clearly visible on process $\mathrm{P} 0$. The applied correction was to correct the iteration counter for the blocking receive.

Please note, that the visualization of an error provides only a first indication of the incorrect behavior. A pre-requisite for correcting the error is identification of the corresponding line of source code. The appropriate feature in MAD is source code connection, which is provided when clicking on the visual representation of an event. A new window opens, displaying event attributes and the filename and line number of the responsible statement.

A different solution to this problem is described in [14]. To avoid the problem of file buffering, shared memory buffers and an external reader process are provided. This way, 
the interesting information can be stored in the tracefiles by a second process, which is not affected by the blocking operation. However, while the benefits of this approach are obvious, its implementation is more difficult than turning on trace file flushing.

In addition to isolated receives, ATEMPT is also able to automatically detect other anomalies in communication patterns. This includes isolated send events or events with different message length as in the following example:

Situation 2: The results of the application contained incorrect values if the number of elements in a particular vector was not divisible by an integer.

In this case, the automatic detection of ATEMPT revealed the incorrect communication pair even before the incorrect results have been verified. Figure 2 presents an example of a communication pair, where the amount of data provided by the sender process $\mathrm{P} 1$ does not fit into the message buffer at the receiver P0. Following the receive on $\mathrm{P} 0$, the process is missing some data and possibly accessing incorrect message buffers.

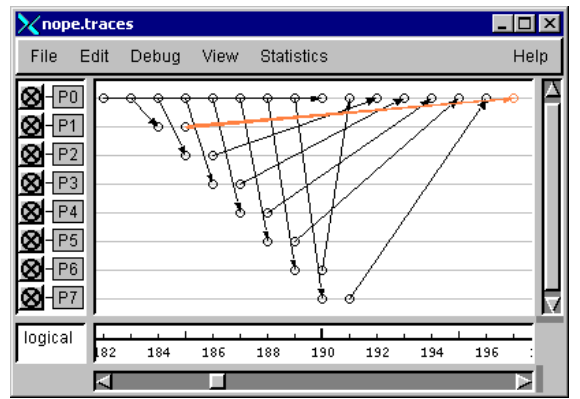

Fig. 2. Different message length at sender and receiver.

The automatic detection of communication errors as described above revealed most MPI related errors in the program with a few executions and correction cycles. We believe, that the benefits of these simple techniques are most usable during debugging.

\section{Advanced Debugging Techniques - Array Visualization}

Besides errors in communication statements, parallelization of a program often involves semantic changes to the program's behavior, including partitioning and distribution of data. In this context, a special feature of MAD for debugging is array visualization (similar functionality is provided by the Prism debugger [1]). With array visualization is it possible to graphically display the contents of up to three dimensions of an arbitrary array. The array itself may be distributed across the available processes using High Performance Fortran-like distribution styles (block, cyclic,... ).

Using array visualization requires manual instrumentation to specify the structure of the array local to each process. The monitor NOPE provides dedicated function calls for array visualization. However, due to the number of possibilities for distributing an 
array across multiple processes, these function calls may represent a major obstacle for the in-experienced debugging user.

The most simple approach of instrumenting a program for array visualization is achieved by placing the function call

monARRAYTRACE (void *array, trcArrayInfo info)

The variable array is a pointer to the start address of the local array, while info describes the structure of the local array and its relation to the parts on the other processes. This description includes the variable type of the elements, the number of dimensions and the size of the array in each dimension. The distribution of the array onto the processes is expressed in HPF-like descriptions. Further details can be specified with overlapping areas at process borders.

An example of an array visualization event is shown in the top-left screenshot of Figure 3 After a broadcast from process P0, every process provides its corresponding part of the global matrix as indicated by the line across all processes. By clicking on the array visualization event, an additional window opens and displays the reconstructed global array as shown with the examples in Figure 3

Situation 3: The array visualization feature has been used to display a 3D-array during processing within the target application. Each process provides only the local array, which is then assembled into the global array. The values stored in the array are displayed as a heat-diagram, with different values indicated by different colors.

With the help of the array visualization feature, distribution errors can often be spotted immediately. While the parallel program should usually ensure a smooth gradient across process borders, errors in array limits or processing are often observed.

The top-right screenshot of Figure 3 shows the 3D-array as computed by the application. For illustration and to emphasize the incorrect behavior, we changed the source code to store a simple function into the parts of the array distributed across all processes. As shown in the middle-left screenshot, the computation of the distributed array seems to be wrong. Stripes along dimension $x$ indicate, that the user did not specify the correct array dimensions on each process. This is underlined by the distribution map of the array as shown in the middle-right screenshot. The global array is distributed across the 8 processes blockwise along dimension $z$.

The bottom-left screenshot of Figure 3 displays the global array after correcting the array boundaries on each process. The disturbance at the process borders has been removed and the computation seems correct. In order to further investigate the 3D-array, mechanisms for modifying the array visualization are provided. An example is shown in the bottom-right screenshot, where a slice of the array along dimension $z$ has been removed in order to display the inside of the matrix.

The array visualization proved a valuable tool during the course of debugging. While the instrumentation of arrays is certainly too difficult for the in-experienced users, the graphical display of distributed data structures represents a huge benefit. Achieving the same knowledge with print statements is certainly a much tougher task. 


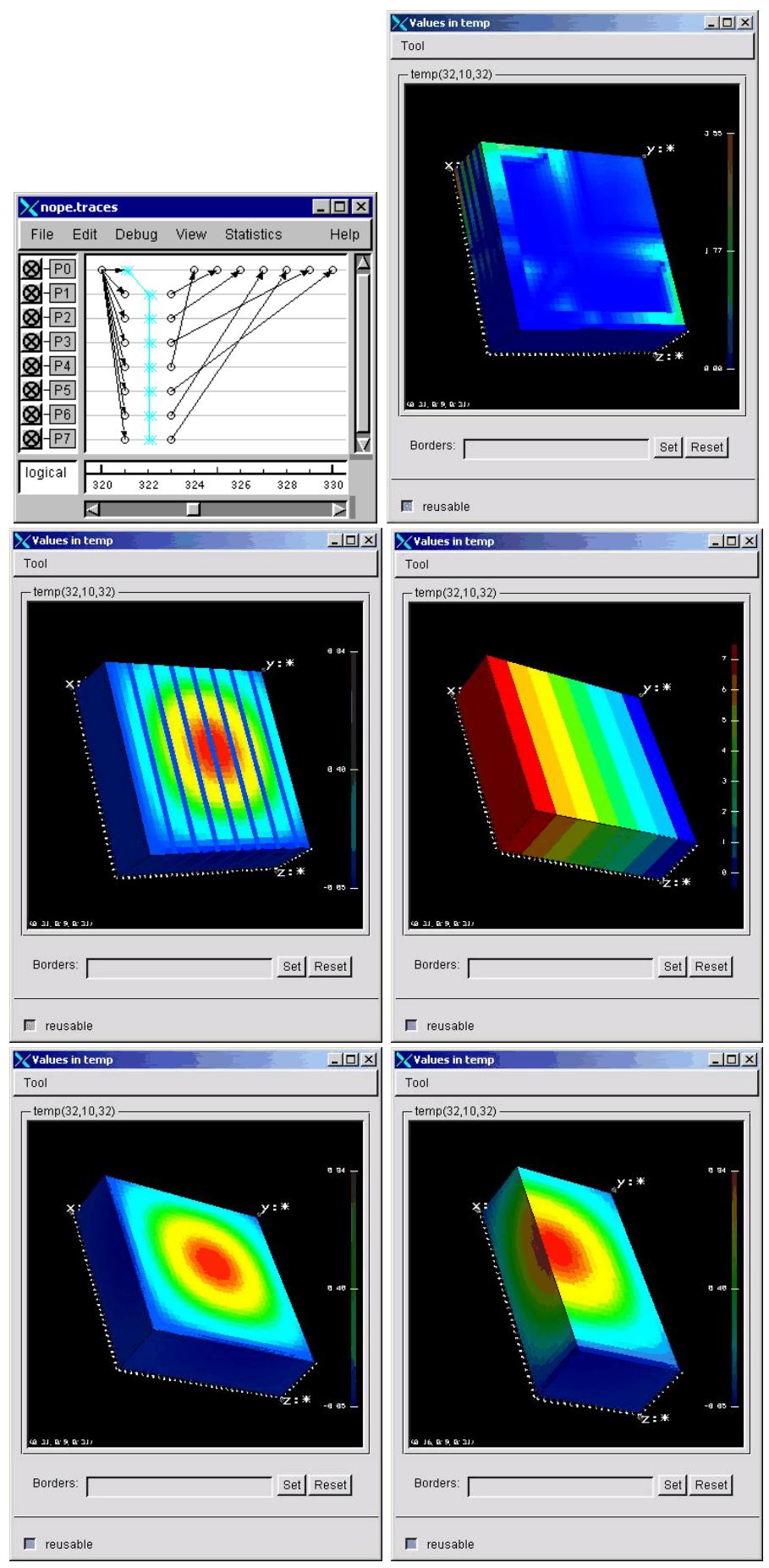

Fig. 3. Examples of array visualization. 


\section{Conclusions and Future Work}

Debugging parallel and distributed CSE applications is a difficult task, which requires dedicated support from software tools. By applying the MAD environment for a realworld application, several discrepancies in viewpoints from the tool developer to the application programmer occurred. While tool developers seem to be interested in solving the most complex problems of program debugging, application programmers seem to be dissatisfied with the functionality of available debugging tools. In fact, the common practice of using print statements for debugging seems to arise from the simplicity of the approach.

On the positive side of our experience with real-world applications, several features of MAD proved vital during the debugging process. The automatic analysis features revealed a series of minor bugs in the code, which were easily traced back based on the space-time diagram and its integrated source code connection. Among the advanced analysis techniques, the verification of data distribution routines with array visualization provided substantial benefits, even though the instrumentation was sometimes tricky.

While debugging the theoretical physics application is now closed, several improvements of the MAD environment are being planned based on the insights provided by this work. On one hand, the automatic analysis activities will be enhanced by pattern analysis, which is expected to reveal repeated communication patterns or minor deviations thereof. On the other hand, the array visualization work will be extended to include additional distributed data structures and their visualization, as well as to simplify the instrumentation process.

Acknowledgments. This work has been supported by a series of colleagues at the Johannes Kepler University, including Prof. Jens Volkert and Prof. Eckhard Krotschek. We gratefully acknowledge J. Messner from the Zentraler Informatikdienst (ZID) of the Johannes Kepler Universität Linz for providing part of his thesis to the MAD project and for assistance in the course of the debugging procedure described in this paper.

\section{References}

1. Allen, D., Bowker, R., Jourdenais, K., Simons, J., Sistare, S., and Title, R.: "The Prism Programming Environment", Proc. Supercomputer Debugging Workshop 91, Albuquerque, New Mexico, USA, pp. 1-7 (November 1991).

2. Campbell, C.E., Krotscheck, E., and Saarela, M.: "Quantum Sticking, Scattering, and Transmission of ${ }^{4} \mathrm{He}$ atoms from Superfluid ${ }^{4} \mathrm{He}$ Surfaces" Physical Review Letters Vol. 80, No. 10, pp. 2169-2172 (1998)

3. Clements, B.E., Krotscheck, E., and Tymczak, C.J.: "Multiphonon excitations in boson quantum films" Physical Review B Vol. 53, No. 18, pp. 12253-12275 (1996)

4. Cunha, J.C., Lourenco, J.M., Antao, T.: "An Experiment in Tool Integration: the DDBG Parallel and Distributed Debugger", EUROMICRO Journal of Systems Architecture, Vol. 45, No. 11, pp. 897-907 (1999).

5. Etnus, LLC: "TotalView Debugger", http://www.etnus.com/Products/TotalView (January 2003) 
6. Hollingsworth, J.K, Miller, B.P., Cargille, J.: "Dynamic Program Instrumentation for Scalable Performance Tools", Proc. SHPCC, 1994 Scalable High Performance Computing Conference, Knoxville, TN, USA, pp. 841-850 (May 1994).

7. Hood, R.: "The p2d2 Project: Building a Portable Distributed Debugger", Proc. SPDT'96, ACM SIGMETRICS Symposium on Parallel and Distributed Tools, Philadelphia, USA, pp. 127-136 (May 1996).

8. Kranzlmüller, D., Grabner, S., Volkert, J.: "Debugging with the MAD Environment", Parallel Computing, Vol. 23, No. 1-2, pp. 199-217 (Apr. 1997)

9. Kranzlmüller, D.: "Event Graph Analysis for Debugging Massively Parallel Programs", PhD Thesis, GUP, Joh. Kepler University Linz, http://www.gup.uni-linz.ac.at/ ${ }^{2 d k / t h e s i s ~(S e p t . ~}$ 2000).

10. Message Passing Interface Forum: "MPI: A Message-Passing Interface Standard - Version 1.1", http://www.mcs.anl.gov/mpi/ (June 1995).

11. Pancake, C.M.: "Visualization Techniques for Parallel Debugging and Performance-Tuning Tools", in: Zomaya, A.Y., " Parallel Computing: Paradigms and Applications", Intl. Thomson Computer Press, pp. 376-393 (1996).

12. Rosenberg, J.B.: "How Debuggers Work: Algorithms, Data Structures, and Architecture", John Wiley \& Sons, New York (1996).

13. Wismüller, R., Oberhuber, M., Krammer, J., and Hansen, O.: "Interactive Debugging and Performance Analysis of Massively Parallel Applications", Parallel Computing, Vol. 22, No. 3, pp. 415-442 (March 1996).

14. Wismüller, R., Dozsa, G., and Drotos, D.: "Using OMIS for On-line Monitoring in the GRADE Programming Environment", Proc. DAPSYS 98, Austrian-Hungarian Workshop on Distributed and Parallel Systems, Budapest, Hungary, pp. 177-184 (Sept. 1998). 